\title{
Improved OFDMA Uplink Transmission via Cooperation in the Presence of Frequency Offsets
}

\author{
Zhongshan Zhang, Chintha Tellambura \\ Department of Electrical and Computer Engineering \\ University of Alberta, Edmonton \\ AB T6G 2V4, Canada \\ \{zszhang, chintha\}@ece.ualberta.ca
}

\author{
Robert Schober \\ Department of Electrical and Computer Engineering \\ University of British Columbia, Vancouver \\ BC V6T 1Z4, Canada \\ rschober@ece.ubc.ca
}

\begin{abstract}
In this paper, we evaluate the performance improvement in an Orthogonal Frequency-Division Multiple Access (OFDMA) uplink achievable with cooperative relaying in the presence of frequency offsets. We consider both the amplifyand-forward (AF) and decode-and-forward (DF) relays. In each transmission, each node plays two roles i.e., the source node and relay, simultaneously, but at different subcarriers. The proposed scheme improves performance without sacrificing the transmission rate and power of the cooperative relays, and the total power used to transmit each symbol, including the power consumed in node the source node and the relays, is kept constant. The outage information rates of the proposed cooperative transmission scheme are derived, and the diversity gain of each forwarding mode in an interference-limited environment is analyzed in this paper. Numerical results illustrate the superior performance of the proposed scheme over the conventional transmission with regard to outage information rate.
\end{abstract}

\section{INTRODUCTION}

Orthogonal Frequency-Division Multiplexing Access (OFDMA) divides the total signal bandwidth into multiple orthogonal subcarrier groups, with each group being allocated to one user [1]. By adaptively allocating and modulating subcarriers for each user, the frequency diversity gain can be improved and, therefore, the OFDMA transmission can be optimized in terms of the bit error rate or the channel capacity [2].

Besides frequency diversity, spatial diversity can also be exploited to improve the diversity gain. One way of realizing the spatial diversity gain is to use "cooperative diversity" [3]. Three time-division-multiple access (TDMA)-based cooperative schemes are proposed in [4], where the relay can operate in either the amplify-and-forward (AF) or decode-andforward (DF) mode. A hybrid forwarding scheme for cooperative relaying in Orthogonal Frequency-Division Multiplex (OFDM)-based networks without considering the frequency offset is proposed in [5]. Both the inter-symbol-interference (ISI) and the inter-carrier-interference (ICI) mitigation in a cooperative space-frequency block coded (SFBC) OFDM by considering the frequency offset is analyzed in [6]. A spacetime cooperative OFDM (CO-OFDM) system in the presence of the frequency offset is proposed in [7], where a diversity gain comparable to that of the conventional space-time coded OFDM system can be achieved in CO-OFDM.
In this paper, performance improvement in an OFDMA uplink via cooperative relaying in the presence of frequency offsets is discussed. As in [8], we also assume that the mobile nodes are able to transmit on one subcarrier at the same time as they listen on another subcarrier. The number of nodes remains fixed during a transmission period but may change across periods. In each transmission, the source node has $M$ relays, and also acts as a relay for the other $M$ nodes simultaneously.

The remainder of this paper is organized as follows. Section II introduces the cooperative OFDMA uplink signal model. The outage information rate of the proposed cooperative transmission is analyzed in Section III. The numerical results are given by Section IV. Finally, Section V concludes the paper.

Notation: $(\cdot)^{H}$ denotes the conjugate transpose of a matrix. The imaginary unit is $\jmath=\sqrt{-1}$. A circularly symmetric complex Gaussian variable with mean $a$ and variance $\sigma^{2}$ is denoted by $z \sim \mathcal{C N}\left(a, \sigma^{2}\right) . \alpha \in \mathcal{A}$ means $\alpha$ is an element of set $\mathcal{A}$. $\mathbf{x}[i]$ represents the $i$-th element of vector $\mathbf{x}$. The $N \times N$ identity matrix is $\mathbf{I}_{N}$, and the $N \times N$ all-zero matrix is $\mathbf{O}_{N}$. $\mathbb{E}\{x\}$ denotes the mean of $x$.

\section{Cooperative OFDMA Uplink Signal Model}

In an OFDMA uplink, each subcarrier of each user is modulated by complex data symbols from a signal constellation such as phase-shift keying (PSK) or quadrature amplitude modulation (QAM). The total number of subcarriers is assumed to be $N$, and $\mathcal{M}$ users are accessing the base station, each user is allocated $N_{u}$ unique subcarriers, where $\mathcal{M} N_{u} \leq N$.

Cooperative transmission among mobile nodes is considered to improve the diversity gain of each node. Each cooperative transmission is performed in two time slots. The time slot is conceptually interchangeable with the OFDMA symbol. In the first time slot, each mobile node transmits its first symbol to all its relays and the base station (node $D$ ) and simultaneously receives the first symbol of the other nodes. In the second time slot, each node will send its second symbol and re-transmit the received symbols from the other nodes simultaneously. Without loss of generality, node $S$ represents the source user and $R_{k}, k \in\{1, \cdots, M\}$ represents the $k$-th relay of $S$, where $M$ is the total number of relays of $S$ in one transmission with $M \leq \mathcal{M}-1$. In different transmissions, $M$ may be different. 
In the second time slot, each node, including $S$, will play two roles simultaneously, i.e., the role of the source node and that of a relay for the other nodes.

Without loss of generality, we assume that each node knows all the channels among all the nodes. Note that quasi-static wireless channels are assumed, and the distance between each pair of mobile nodes is assumed to be not far and that, therefore, only small-scale fading occurs on the channels between them with $H_{a b}^{(i)} \sim \mathcal{C N}(0,1)$ for each $b \neq a, D$. We also assume that the distance between each node and $D$ is large enough to produce large-scale fading with $H_{a D}^{(i)} \sim \mathcal{C N}\left(0, \mathcal{L}_{u}\right)$ for each $a$, where $\mathcal{L}_{u}<1$ is the large-scale fading coefficient.

In each transmission of node $S$, its relays can be chosen as follows: we first set a threshold $\nu$ where $\nu>0$, and node $k$ will act as a relay of $S$ if $\hbar_{k}=\operatorname{trace}\left\{\mathbf{H}_{S R_{k}} \mathbf{H}_{S R_{k}}^{H}\right\} \geq \nu N_{u}$, $1 \leq k \leq \mathcal{M}-1$. From [9, page 41] we know that $\hbar_{k}$ can be approximated as a central chi-square RV with $2 N_{u}$ degrees of freedom, and the probability of $\hbar_{k} \geq \nu N_{u}$ is

$$
P_{\nu}=\mathrm{P}_{\mathrm{r}}\left\{\hbar_{k} \geq \nu N_{u}\right\}=e^{-N_{u} \nu} \sum_{m=0}^{N_{u}-1} \frac{\nu^{m} N_{u}^{m}}{m !},
$$

where $\operatorname{Pr}\{\mathcal{Q}\}$ means the probability that event $\mathcal{Q}$ happens. The probability that the source node has $M$ relays is given by

$$
P_{\nu, M}=\left(\begin{array}{c}
\mathcal{M}-1 \\
M
\end{array}\right) P_{\nu}^{M}\left(1-P_{\nu}\right)^{\mathcal{M}-M-1} .
$$

\section{A. AF Mode}

The transmit-receive relationship in a two-time-slot period is

$$
\mathbf{r}_{S}^{\mathrm{AF}}=\underbrace{\left[\begin{array}{cc}
\mathbf{F}_{S}^{H} \mathbf{V}_{S D, 1} & \mathbf{O} \\
\mathbf{F}_{S}^{H} \mathbf{V}_{S R D, 2}^{\mathrm{AF}} & \mathbf{F}_{S}^{H} \mathbf{V}_{S D, 2}
\end{array}\right]}_{\mathbf{A}_{S}^{\mathrm{AF}}} \underbrace{\left[\begin{array}{c}
\mathbf{x}_{S, 1} \\
\mathbf{x}_{S, 2}
\end{array}\right]}_{\mathbf{x}_{S}}+\underbrace{\left[\begin{array}{c}
\boldsymbol{\xi}_{S, 1}^{\mathrm{AF}} \\
\boldsymbol{\xi}_{S, 2}^{\mathrm{AF}}
\end{array}\right]}_{\boldsymbol{\xi}_{S}^{\mathrm{AF}}},
$$

where $\mathbf{r}_{S}^{\mathrm{AF}}$ is a $2 N \times 1$ receive vector, $\mathbf{V}_{S D, 1}$, $\mathbf{V}_{S D, 2}, \quad \mathbf{V}_{S R D, 2}^{\mathrm{AF}}, \quad \boldsymbol{\xi}_{S, 1}^{\mathrm{AF}}$ and $\boldsymbol{\xi}_{S, 2}^{\mathrm{AF}}$ are defined in (4), $\boldsymbol{\Phi}_{a, 1}=\operatorname{diag}\left\{\sqrt{P_{a, 1, i}}: i \in G_{a}, a \in\left\{S, \cdots, R_{k},\right\}\right\}$ is the $N_{u} \times N_{u}$ transmit power matrix of node $a, \mathbf{H}_{a b}=$ $\operatorname{diag}\left\{H_{a b}^{(i)}: i \in G_{a}, a \in\left\{S, \cdots, R_{k},\right\}, b \in\left\{S, D, \cdots, R_{k},\right\}\right\}$ with $H_{a b}^{(i)}$ denotes the channel attenuation between nodes $a$ and $b$ at the $i$-th subcarrier, and $G_{a}$ stands for the subcarriers allocated to node $a . \boldsymbol{\Phi}_{a, 2}^{t}=\operatorname{diag}\left\{\sqrt{P_{a, 2, i}^{t}}: i \in G_{a}\right\}$ and $\boldsymbol{\Phi}_{a, 2}^{r}=\operatorname{diag}\left\{\sqrt{P_{a, 2, i}^{r}}: i \in G_{a}\right\}\left(a \in\left\{S, R_{1}, \cdots, R_{M}\right\}\right)$ represent the power allocation to node $a$ used to transmit its own signal and retransmit signals for the other nodes in the second time slot, respectively. In this paper, we assume that each mobile node does not know the uplink channel, and that identical power allocation in each subcarrier is performed in either $S \rightarrow D$ or $R \rightarrow D$ transmission.

In the first time slot, $P_{a, 1, i}=\bar{P}$ for each $a$ and $i$, and that in the second time slot, by allocating $\alpha N_{u} \bar{P}$ to transmit its second symbol, the source node will also use the remaining $(1-\alpha) N_{u} \bar{P}$ to retransmit signals for its $M$ relays, where $0 \leq \alpha \leq 1$. Therefore, we have $P_{a, 2, i}^{t}=\alpha \bar{P}$ and $P_{a, 2, i}^{r}=\frac{(1-\alpha) \bar{P}}{M}$. The matrix $\mathbf{E}_{a b}$ is defined as $\mathbf{E}_{a b}=$ $\operatorname{diag}\left\{e^{\jmath \psi_{a b}}, \cdots, e^{\jmath\left(\frac{2 \pi \varepsilon_{a b}(N-1)}{N}+\psi_{a b}\right)}\right\}$ with $\psi_{a b}$ and $\varepsilon_{a b}$ representing the initial phase and normalized carrier frequency offset (the frequency offset normalized to one subcarrier bandwidth) between nodes $a$ and $b$, respectively. ${ }^{1}$ We assume that each $\varepsilon_{a D}, a \in\left\{S, R_{1}, \cdots, R_{M}\right\}$, is an independent and identically distributed (i.i.d.) random variable (RV) with zero mean and variance $\sigma_{\epsilon}^{2}$ (and is not necessarily Gaussian) and, therefore, we have $\operatorname{Var}\left\{\varepsilon_{a D}\right\}=\sigma_{\epsilon}^{2}$ and $\operatorname{Var}\left\{\varepsilon_{a b}\right\}=2 \sigma_{\epsilon}^{2}$, $a, b \in\left\{S, R_{1}, \cdots, R_{M}\right\}, a \neq b$. We also have $\varepsilon_{S D}=$ $\varepsilon_{S R_{k}}+\varepsilon_{R_{k} D}$ for each $k . \mathbf{F}_{a}$ is the $N \times N_{u}$ matrix, which denotes the Inverse Discrete Fourier Transform (IDFT) matrix for the $a$-th user. $\mathbf{F}_{a}$ can be generated from $\mathbf{F}$ by deleting all the columns with the column indexes not belonging to $G_{a}$, where $\mathbf{F}$ is a $N \times N$ matrix with $[\mathbf{F}]_{m n}=\frac{1}{\sqrt{N}} e^{\frac{j 2 \pi m n}{N}}, \quad 0 \leq$ $m, n \leq N-1$, and $N$ is the Discrete Fourier Transform (DFT) length. $\mathbf{x}_{a, i}$ is the $N_{u} \times 1$ transmit vector of user $a$ at the $i$-th time slot, and without loss of generality, $\mathbf{x}_{a, i}[\mathrm{~m}]$ are i.i.d. complex RVs with zero mean and unit variance. $\mathbf{w}_{a}$ and $\mathbf{w}_{D, i}$ are vectors of additive white Gaussian noise (AWGN) with $\left\{\mathbf{w}_{a}[m], \mathbf{w}_{D, i}[m]: a \in\left\{S, R_{1}, \cdots, R_{M}\right\}, i=(1,2)\right\} \sim$ $\mathcal{C N}\left(0, \sigma_{w}^{2}\right) \cdot \varpi_{a b}=\left(\theta_{\nu} \bar{P}+\sigma_{w}^{2}\right)^{-\frac{1}{2}}$ represents the amplify coefficients at node $a$ when it retransmits the signal for node $b$, where $\theta_{\nu}=1+\left(N_{u} ! \sum_{m=0}^{N_{u}-1} \frac{\nu^{m-N_{u}} N_{u}^{m-N_{u}}}{m !}\right)^{-1}$.

\section{B. DF Mode}

In the first time slot, the transmit-receive relationship of the DF mode is identical to that of the AF mode. However, in the second time slot, before retransmission, each relay node in the DF mode should first demodulate and decode the signal received from the other cooperative nodes. ${ }^{2}$ The transmitreceive relationship is

$$
\mathbf{r}_{S}^{\mathrm{DF}}=\underbrace{\left[\begin{array}{cc}
\mathbf{F}_{S}^{H} \mathbf{V}_{S D, 1} & \mathbf{O} \\
\mathbf{F}_{S}^{H} \mathbf{V}_{S R D, 2}^{\mathrm{DF}} & \mathbf{F}_{S}^{H} \mathbf{V}_{S D, 2}
\end{array}\right]}_{\mathbf{A}_{S}^{\mathrm{DF}}} \underbrace{\left[\begin{array}{c}
\mathbf{x}_{S, 1} \\
\mathbf{x}_{S, 2}
\end{array}\right]}_{\mathbf{x}_{S}}+\underbrace{\left[\begin{array}{c}
\boldsymbol{\xi}_{S}^{\mathrm{DF}} \\
\boldsymbol{\xi}_{S, 2}^{\mathrm{DF}}
\end{array}\right]}_{\boldsymbol{\xi}_{S}^{\mathrm{DF}}},
$$

where $\mathbf{V}_{S R D, 2}^{\mathrm{DF}}, \boldsymbol{\xi}_{S, 1}^{\mathrm{DF}}, \boldsymbol{\xi}_{S, 2}^{\mathrm{DF}}$ and $\boldsymbol{\eta}_{S}^{\mathrm{DF}}$ are defined in (6).

Unlike the AF mode, since the interference generated in the $S \rightarrow R$ transmission is eliminated in the DF mode, the effective Signal-to-Interference-plus-Noise Ratio (SINR) in the DF mode is improved considerably, but at the cost of an additional complexity in hardware design in each relay.

\footnotetext{
${ }^{1}$ Without loss of generality, we assume that each $\psi_{a b}$ has been estimated and corrected, i.e., $\psi_{a b}=0$ is assumed in the following sections.

${ }^{2}$ The relay node can demode and decode its relay signals correctly if and only if the transmit rate of each node does not exceed the corresponding capacity of each $S \rightarrow R$ channel.
} 


$$
\begin{aligned}
& \mathbf{V}_{S D, 1}= \mathbf{E}_{S D} \mathbf{F}_{S} \mathbf{H}_{S D} \boldsymbol{\Phi}_{S, 1}, \quad \mathbf{V}_{S D, 2}=\mathbf{E}_{S D} \mathbf{F}_{S} \mathbf{H}_{S D} \boldsymbol{\Phi}_{S, 2}^{t}, \\
& \mathbf{V}_{S R D, 2}^{\mathrm{AF}}= \sum_{k=1}^{M} \varpi_{R_{k} S} \mathbf{E}_{R_{k} D} \mathbf{F}_{S} \mathbf{H}_{R_{k} D} \boldsymbol{\Phi}_{R_{k}, 2}^{r} \mathbf{F}_{S}^{H} \mathbf{E}_{S R_{k}} \mathbf{F}_{S} \mathbf{H}_{S R_{k}} \boldsymbol{\Phi}_{S, 1}, \quad \boldsymbol{\xi}_{S, 1}^{\mathrm{AF}}=\mathbf{F}_{S}^{H}\left(\sum_{k=1}^{M} \mathbf{E}_{R_{k} D} \mathbf{F}_{R_{k}} \mathbf{H}_{R_{k} D} \boldsymbol{\Phi}_{R_{k}, 1} \mathbf{x}_{R_{k}, 1}+\mathbf{w}_{D, 1}\right), \\
& \boldsymbol{\xi}_{S, 2}^{\mathrm{AF}}=\mathbf{F}_{S}^{H}\left[\sum_{k=1}^{M}\left(\mathbf{V}_{R_{k} S D, 2}^{\mathrm{AF}} \mathbf{x}_{R_{k}, 1}+\sum_{l=1, l \neq k}^{M} \mathbf{V}_{R_{l} R_{k} D, 2}^{\mathrm{AF}} \mathbf{x}_{R_{l}, 1}+\mathbf{V}_{R_{k} D, 2} \mathbf{x}_{R_{k}, 2}\right)+\tilde{\mathbf{w}}_{D, 2}\right], \quad \mathbf{V}_{R_{k} S D, 2}^{\mathrm{AF}}=\varpi_{R_{k} S} \mathbf{E}_{S D} \mathbf{F}_{R_{k}} \mathbf{H}_{S D} \boldsymbol{\Phi}_{S, 2}^{r} \mathbf{F}_{R_{k}}^{H} \mathbf{V}_{R_{k} S}, \\
& \mathbf{V}_{R_{k} D, 2}=\mathbf{E}_{R_{k} D} \mathbf{F}_{R_{k}} \mathbf{H}_{R_{k} D} \boldsymbol{\Phi}_{R_{k}, 2}^{t}, \quad \mathbf{V}_{R_{l} R_{k} D, 2}^{\mathrm{AF}}=\varpi_{R_{l} R_{k}} \mathbf{E}_{R_{k} D} \mathbf{F}_{R_{l}} \mathbf{H}_{R_{k} D} \boldsymbol{\Phi}_{R_{k}, 2}^{r} \mathbf{F}_{R_{l}}^{H} \mathbf{E}_{R_{l} R_{k}} \mathbf{F}_{R_{l}} \mathbf{H}_{R_{l} R_{k}} \boldsymbol{\Phi}_{R_{l}, 1}, \\
& \tilde{\mathbf{w}}_{D, 2}= \sum_{k=1}^{M} \varpi_{R_{k} S} \mathbf{E}_{S D} \mathbf{F}_{R_{k}} \mathbf{H}_{S D} \boldsymbol{\Phi}_{S, 2}^{r} \mathbf{F}_{R_{k}}^{H} \mathbf{w}_{S}+\sum_{k=1}^{M} \varpi_{S R_{k}} \mathbf{E}_{R_{k} D} \mathbf{F}_{S} \mathbf{H}_{R_{k} D} \boldsymbol{\Phi}_{R_{k}, 2}^{r} \mathbf{F}_{S}^{H} \mathbf{w}_{R_{k}} \\
&+\sum_{k=1}^{M} \sum_{l=1, l \neq k}^{M} \varpi_{R_{l} R_{k}} \mathbf{E}_{R_{k} D} \mathbf{F}_{R_{l}} \mathbf{H}_{R_{k} D} \boldsymbol{\Phi}_{R_{k}, 2}^{r} \mathbf{F}_{R_{l}}^{H} \mathbf{w}_{R_{k}}+\mathbf{w}_{D, 2},
\end{aligned}
$$

$$
\begin{aligned}
& \mathbf{V}_{S R D, 2}^{\mathrm{DF}}=\sum_{k=1}^{M} \mathbf{E}_{R_{k} D} \mathbf{F}_{S} \mathbf{H}_{R_{k} D} \boldsymbol{\Phi}_{R_{k}, 2}^{r}, \quad \boldsymbol{\xi}_{S, 1}^{\mathrm{DF}}=\mathbf{F}_{S}^{H}\left(\sum_{k=1}^{M} \mathbf{V}_{R_{k} D, 1} \mathbf{x}_{R_{k}, 1}+\mathbf{w}_{D, 1}\right), \quad \boldsymbol{\xi}_{S, 2}^{\mathrm{DF}}=\mathbf{F}_{S}^{H} \boldsymbol{\eta}_{S}^{\mathrm{DF}}+\mathbf{F}_{S}^{H} \mathbf{w}_{D, 2} \\
& \boldsymbol{\eta}_{S}^{\mathrm{DF}}=\sum_{k=1}^{M} \mathbf{E}_{S D} \mathbf{F}_{R_{k}} \mathbf{H}_{S D} \boldsymbol{\Phi}_{S, 2}^{r} \mathbf{x}_{R_{k}, 1}+\sum_{k=1}^{M} \sum_{l=1, l \neq k}^{M} \mathbf{E}_{R_{k} D} \mathbf{F}_{R_{l}} \mathbf{H}_{R_{k} D} \boldsymbol{\Phi}_{R_{k}, 2}^{r} \mathbf{x}_{R_{l}, 1}+\sum_{k=1}^{M} \mathbf{E}_{R_{k} D} \mathbf{F}_{R_{k}} \mathbf{H}_{R_{k} D} \boldsymbol{\Phi}_{R_{k}, 2}^{t} \mathbf{x}_{R_{k}, 2}
\end{aligned}
$$

\section{OUtAGE INFORMATION RATE IMPROVEMENT BY USING COOPERATIVE TRANSMISSION}

In this section, we will derive a closed-form representation of the outage information rate in a cooperative OFDMA uplink transmission in the presence of frequency offsets.

\section{A. Outage Information Rate in the AF Mode}

For a given $M$, the $p(0 \leq p \leq 1)$ outage information rate of the proposed cooperative transmission with $\mathrm{AF}$ relays, $\mathcal{I}_{p, \alpha, M}^{\mathrm{AF}}$, is defined as

$$
\mathrm{P}_{\mathrm{r}}\left\{\mathcal{I}_{M}^{\mathrm{AF}}\left(\mathbf{x}_{S} ; \mathbf{r}_{S}^{\mathrm{AF}} \mid \varepsilon_{z k}, \mathbf{H}_{z k}\right) \leq \mathcal{I}_{p, \alpha, M}^{\mathrm{AF}}\right\}=p
$$

where $\mathcal{I}_{M}^{\mathrm{AF}}\left(\mathbf{x}_{S} ; \mathbf{r}_{S}^{\mathrm{AF}} \mid \varepsilon_{z k}, \mathbf{H}_{z k}\right), \mathbf{G}_{S}^{\mathrm{AF}}, \boldsymbol{\mu}_{S, 1}$ and $\boldsymbol{\mu}_{S, 2}$ are defined in (8).

When $0 \leq \alpha \leq 1$, the averaged outage information rate is $\overline{\mathcal{I}_{\text {out }}^{\mathrm{AF}}}=\sum_{M=1}^{\mathcal{M}-1} P_{\nu, M} \mathcal{I}_{p, \alpha, M}^{\mathrm{AF}}$, where $\mathcal{I}_{p, \alpha, M}^{\mathrm{AF}}, \mathcal{Z}_{\alpha, M}^{A F}, \sigma_{\boldsymbol{\xi}_{S, 1}^{\mathrm{AF}}}^{2}$ and $\sigma_{\boldsymbol{\xi}_{S, 2}^{\mathrm{AF}}}^{2}$ are defined in (9), and $\beta_{1}=1-\frac{\pi^{2} \sigma_{\epsilon}^{2}}{3}+\frac{\pi^{4} \sigma_{\epsilon}^{4}}{20}$.

\section{B. Outage Information Rate in the DF Mode}

The channel information rate of the DF mode with a cooperative relay is different from that of the AF mode. Without loss of generality, we assume that the transmission rate of node $S$ in the first time slot is $\rho_{1}$. For a given $M$ and assuming that the relays are able to decode $\mathbf{x}_{S, 1}$ correctly, $\rho_{1}$ should satisfy $\rho_{1} \leq \rho_{1}^{*}=\min \left\{\rho_{1,1}, \cdots, \rho_{1, M}\right\}$, where $\rho_{1, k}=\log _{2} \operatorname{det}\left(\mathbf{I}_{N_{u}}+\mathbf{F}_{S}^{H} \mathbf{V}_{S R_{k}} \mathbf{V}_{S R_{k}}^{H} \mathbf{F}_{S}\right)$. In the second time slot, the uplink transmission can be formulated as a typical multiple-access channel (MAC). We can define the transmission rate constraint in the second time slot as

$$
\begin{aligned}
& \rho_{1} \leq \min \left\{\log _{2} \operatorname{det}\left(\mathbf{I}_{N_{u}}+\mathbf{Z}_{1}\right), \rho_{1}^{*}\right\} \\
& \rho_{2} \leq \log _{2} \operatorname{det}\left(\mathbf{I}_{N_{u}}+\mathbf{F}_{S}^{H} \mathbf{V}_{S D, 2} \mathbf{V}_{S D, 2}^{H} \mathbf{F}_{S}\right), \\
& \rho_{1}+\rho_{2} \leq \log _{2} \operatorname{det}\left(\mathbf{I}_{2 N_{u}}+\mathbf{Z}^{\mathrm{DF}}\right),
\end{aligned}
$$

where $\mathbf{Z}_{1}=\mathbf{F}_{S}^{H}\left(\mathbf{V}_{S R D, 2}^{\mathrm{DF}}\left(\mathbf{V}_{S R D, 2}^{\mathrm{DF}}\right)^{H}+\mathbf{V}_{S D, 1} \mathbf{V}_{S D, 1}^{H}\right) \mathbf{F}_{S}$, $\mathbf{Z}^{\mathrm{DF}}=\left(\mathbf{G}_{S}^{\mathrm{DF}}\right)^{-\frac{1}{2}} \mathbf{A}_{S}^{\mathrm{DF}}\left(\mathbf{A}_{S}^{\mathrm{DF}}\right)^{H}\left(\mathbf{G}_{S}^{\mathrm{DF}}\right)^{-\frac{H}{2}}, \mathbf{G}_{S}^{\mathrm{DF}}=$
$\left[\begin{array}{c}\boldsymbol{\mu}_{S, 1}+\sigma_{w}^{2} \mathbf{I}_{N_{u}} \\ \boldsymbol{\mu}_{S, 2}+\sigma_{w}^{2} \mathbf{I}_{N_{u}}\end{array}\right]\left[\begin{array}{c}\boldsymbol{\mu}_{S, 1}+\sigma_{w}^{2} \mathbf{I}_{N_{u}} \\ \boldsymbol{\mu}_{S, 2}+\sigma_{w}^{2} \mathbf{I}_{N_{u}}\end{array}\right]^{H} \cdot$ The mutual
information of the DF mode is given by $\mathcal{I}_{M}^{\mathrm{DF}}\left(\mathbf{x}_{S} ; \tilde{\mathbf{r}}_{S}^{\mathrm{DF}}\right)=\frac{1}{2} \min \left\{\rho_{1}+\rho_{2}, \quad \log _{2} \operatorname{det}\left(\mathbf{I}_{2 N_{u}}+\mathbf{Z}^{\mathrm{DF}}\right)\right\}$.

When $0 \leq \alpha \leq 1$, the averaged outage information rate is given by $\overline{\mathcal{I}_{\text {out }}^{\mathrm{DF}}}=\sum_{M=1}^{\overline{\mathcal{M}}-1} P_{\nu, M} \mathcal{I}_{p, \alpha, M}^{\mathrm{DF}}$, where $\mathcal{I}_{p, \alpha, M}^{\mathrm{DF}}, \beta_{2}, \mathcal{Z}_{\alpha, M, \mathrm{r}}^{D F}$ and $\mathcal{Z}_{\alpha, M}^{D F}$ are defined in (12).

\section{Numerical Results}

In our simulation, a wireless channel of OFDMA uplink transmission with a bandwidth of $10 \mathrm{MHz}$ and $N=1024$ is considered. A cyclic-prefix of length 64 is padded to the front of each symbol. A total of 64 users are assumed to access the base station.

The outage information rate is a function of $\nu$, and the optimal outage information rates are obtained as $\overline{\mathcal{I}_{\text {out }}^{\mathrm{AF}}}=\max _{\nu} \mathcal{I}_{\text {out }}^{\mathrm{AF}}$ and $\overline{\mathcal{I}_{\text {out }}^{\mathrm{DF}}}=\max _{\nu} \mathcal{I}_{\text {out }}^{\mathrm{DF}}$, for the $\mathrm{AF}$ and DF modes, respectively. $\overline{\mathcal{I}_{\text {out }}^{\mathrm{AF}}}$ and $\overline{\mathcal{I}_{\text {out }}^{\mathrm{DF}}}$ as functions of $\nu$ are illustrated in Fig. 1. It is shown that the DF mode does not always outperform the AF mode for each $\alpha$. We can explain it as follows: in 


$$
\begin{aligned}
& \mathcal{I}_{M}^{\mathrm{AF}}\left(\mathbf{x}_{S} ; \mathbf{r}_{S}^{\mathrm{AF}} \mid \varepsilon_{z k}, \mathbf{H}_{z k}\right)=\frac{1}{2} \log _{2} \operatorname{det}\left(\mathbf{I}_{2 N_{u}}+\left(\mathbf{G}_{S}^{\mathrm{AF}}\right)^{-\frac{1}{2}} \mathbf{A}_{S}^{\mathrm{AF}}\left(\mathbf{A}_{S}^{\mathrm{AF}}\right)^{H}\left(\mathbf{G}_{S}^{\mathrm{AF}}\right)^{-\frac{H}{2}}\right), \quad \boldsymbol{\mu}=\left[\begin{array}{c}
\boldsymbol{\mu}_{S, 2}+\left[\frac{\mathcal{L}_{u}(M+1)(1-\alpha) \bar{P}}{\theta_{\nu} \bar{P}+\sigma_{w}^{2}}+1\right] \sigma_{w}^{2} \mathbf{I}_{N_{u}}
\end{array}\right] \\
& \mathbf{G}_{S}^{\mathrm{AF}}=\boldsymbol{\mu} \boldsymbol{\mu}^{H}, \quad \boldsymbol{\mu}_{S, 1}=\mathbf{F}_{S}^{H} \sum_{k=1}^{M} \mathbf{V}_{R_{k} D, 1} \mathbf{x}_{R_{k}, 1}, \quad \boldsymbol{\mu}_{S, 2}=\mathbf{F}_{S}^{H} \sum_{k=1}^{M}\left[\left(\mathbf{V}_{R_{k} S D, 2}^{\mathrm{AF}}+\mathbf{V}_{R_{k} D, 2}\right) \mathbf{x}_{R_{k}, 1}+\sum_{l=1, l \neq k}^{M} \mathbf{V}_{R_{l} R_{k} D, 2}^{\mathrm{AF}} \mathbf{x}_{R_{l}, 1}\right] .
\end{aligned}
$$

$$
\begin{aligned}
& \mathcal{I}_{p, \alpha, M}^{\mathrm{AF}}=\frac{N_{u}}{2} \log _{2}\left(\mathcal{Z}_{\alpha, M}^{A F}+\frac{\mathcal{L}_{u} \cdot \theta_{\nu}(1-\alpha) \bar{P}^{2} \beta_{1}(p \cdot M !) \frac{1}{M}}{M \sigma_{\boldsymbol{\xi}_{S, 2}^{\mathrm{AF}}}^{2}\left(\theta_{\nu} \bar{P}+\sigma_{w}^{2}\right)}\right), \quad \mathcal{Z}_{\alpha, M}^{A F}=\left(1+\frac{\alpha \cdot \mathcal{L}_{u} \cdot \bar{P} \cdot \beta_{1} \cdot \ln \frac{1}{1-p}}{\sigma_{\boldsymbol{\xi}_{S, 2}^{\mathrm{AF}}}^{2}}\right)\left(1+\frac{\mathcal{L}_{u} \cdot \bar{P} \cdot \beta_{1} \cdot \ln \frac{1}{1-p}}{\sigma_{\boldsymbol{\xi}_{S, 1}^{\mathrm{AF}}}^{2}}\right), \\
& \sigma_{\boldsymbol{\xi}_{S, 1}^{\mathrm{AF}}}^{2}=\frac{\mathcal{L}_{u} M \pi^{2} \sigma_{\epsilon}^{2} \bar{P}}{3 \mathcal{M}}+\sigma_{w}^{2}, \quad \sigma_{\boldsymbol{\xi}_{S, 2}^{\mathrm{AF}}}^{2}=\frac{2 \mathcal{L}_{u} \theta_{\nu}\left(M^{2}-M+1\right)(1-\alpha) \pi^{2} \sigma_{\epsilon}^{2} \bar{P}^{2}+\mathcal{L}_{u} \alpha M \pi^{2} \sigma_{\epsilon}^{2} \bar{P}}{3 \mathcal{M}}+\left(\mathcal{L}_{u}(M+1)(1-\alpha) \bar{P}+1\right) \sigma_{w}^{2} .
\end{aligned}
$$

$$
\begin{aligned}
& \mathcal{I}_{p, \alpha, M}^{\mathrm{DF}}=\frac{N_{u}}{2} \times \log _{2} \min \left\{\mathcal{Z}_{\alpha, M, \mathrm{r}}^{D F}, \mathcal{Z}_{\alpha, M}^{D F}+\frac{\mathcal{L}_{u}(1-\alpha) \bar{\gamma} \beta_{1}(p \cdot M !) \frac{1}{M}}{\frac{\mathcal{L}_{u} M^{2} \pi^{2} \sigma_{\epsilon}^{2} \bar{\gamma}}{3 \mathcal{M}}+M}\right\}, \quad \beta_{2}=1-\frac{2 \pi^{2} \sigma_{\epsilon}^{2}}{3}+\frac{\pi^{4} \sigma_{\epsilon}^{4}}{5}, \\
& \mathcal{Z}_{\alpha, M, \mathrm{r}}^{D F}=\left(1+\frac{\alpha \cdot \mathcal{L}_{u} \cdot \bar{\gamma} \cdot \beta_{1} \cdot \ln \frac{1}{1-p}}{\frac{\mathcal{L}_{u} M \pi^{2} \sigma_{\epsilon}^{2} \bar{\gamma}}{3 \mathcal{M}}+1}\right)\left(1+\frac{\theta_{\nu} \cdot \bar{\gamma} \cdot \beta_{2} \cdot \ln \frac{1}{1-p}}{\frac{2(M-1) \pi^{2} \sigma_{\epsilon}^{2} \bar{\gamma}}{3 \mathcal{M}}+1}\right), \quad \mathcal{Z}_{\alpha, M}^{D F}=\left(1+\frac{\alpha \cdot \mathcal{L}_{u} \cdot \bar{\gamma} \cdot \beta_{1} \cdot \ln \frac{1}{1-p}}{\frac{\mathcal{L}_{u} M \pi^{2} \sigma_{\epsilon}^{2} \bar{\gamma}}{3 \mathcal{M}}+1}\right)\left(1+\frac{\mathcal{L}_{u} \cdot \bar{\gamma} \cdot \beta_{1} \cdot \ln \frac{1}{1-p}}{\frac{\mathcal{L}_{u} M \pi^{2} \sigma_{\epsilon}^{2} \bar{\gamma}}{3 \mathcal{M}}+1}\right) .
\end{aligned}
$$

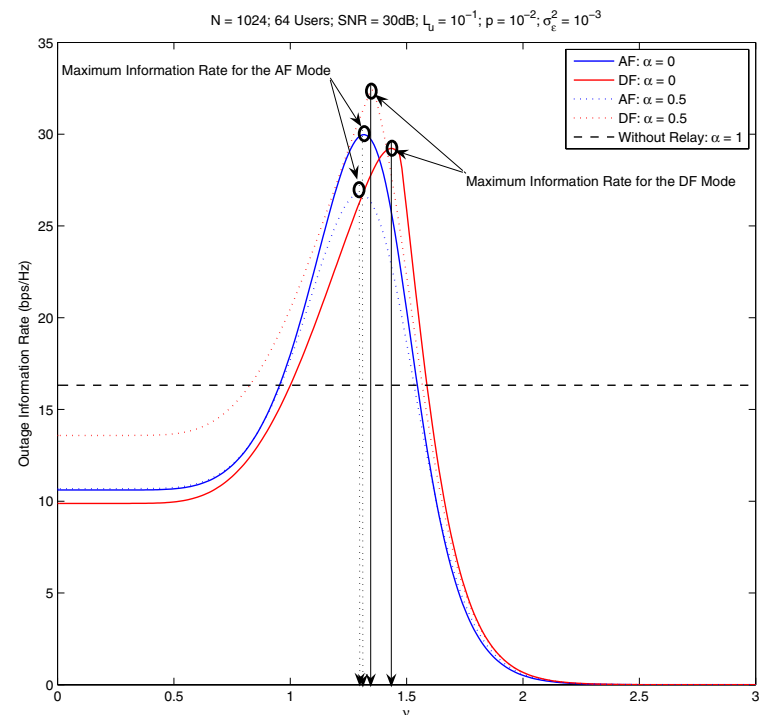

Fig. 1. Outage information rate as a function of $\nu$.

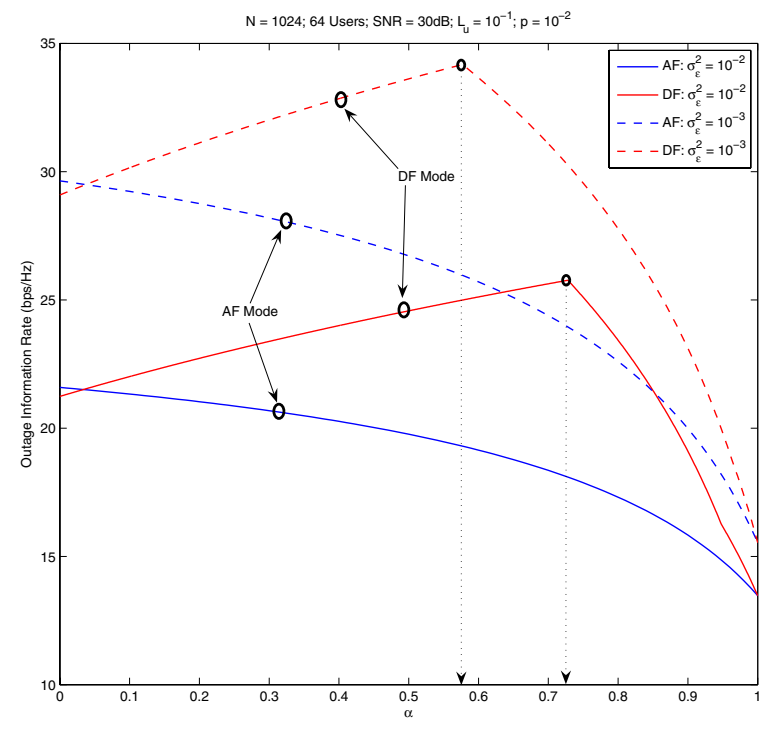

Fig. 2. Outage information rate as a function of $\alpha$. an interference-limited environment, the transmission rates of both $S \rightarrow D$ and $S \rightarrow R$ links are functions of $\sigma_{\epsilon}^{2}$. When $\alpha$ is very small, the transmission rate of each mode is dominated by $S \rightarrow R \rightarrow D$ link. Since $\operatorname{Var}\left\{\varepsilon_{S R}\right\}=2 \cdot \operatorname{Var}\left\{\varepsilon_{S D}\right\}$ for each $R$, the large demodulate errors at node $R$ will degrade the information rate of the DF mode.

The outage information rate as a function of $\alpha$ is illustrated in Fig. 2. For a given SNR and $p$, the outage information rate of the AF mode is a monotonically decreasing function of $\alpha$. Different from it, for the DF mode, as $\alpha$ increases, the outage information rate first increases to its maximum value, and then decreases if $\alpha$ continuously increases. For a given $\sigma_{\epsilon}^{2}$, the DF mode outperforms the AF mode only when $\alpha$ is larger than a specified threshold. For example, when $\sigma_{\epsilon}^{2}=10^{-2}$, this threshold is $\alpha=0.034$. This threshold for $\sigma_{\epsilon}^{2}=10^{-3}$ is $\alpha=$ 0.037. Numerical results in Fig. 2 show that in an interferencelimited environment, cooperative transmission with DF relays achieves a relatively better performance than cooperations with AF relays, if $\alpha$ is larger than a specified threshold.

The outage information rate of the proposed cooperative transmission as a function of $\sigma_{\epsilon}^{2}$ is illustrated in Fig. 3. Note that in this simulation, $\overline{\mathcal{I}_{\text {out }}^{\mathrm{AF}}}$ and $\overline{\mathcal{I}_{\text {out }} \mathrm{DF}}$ that jointly optimized in 


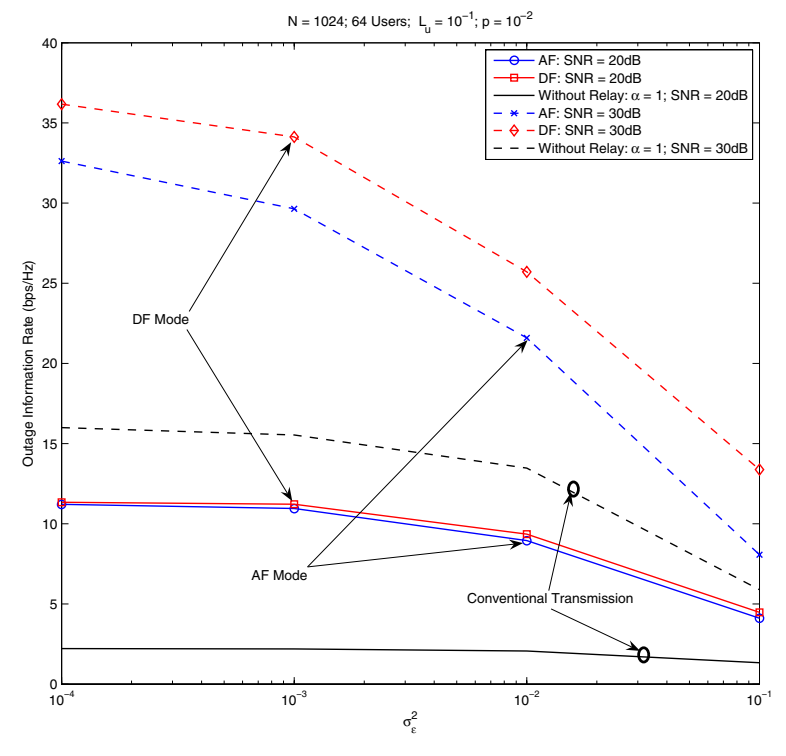

Fig. 3. Outage information rate as a function of $\sigma_{\epsilon}^{2}$.

terms of $(\nu, \alpha)$ are compared. For each $\sigma_{\epsilon}^{2}$, the cooperative transmission with each mode will always outperform the conventional transmission in terms of the outage information rate, and a smaller $\sigma_{\epsilon}^{2}$ implies a higher performance improvement. For example, when SNR $=20 \mathrm{~dB}$, the outage information rate improvement of the $\mathrm{AF}$ mode (or the $\mathrm{DF}$ mode) over the conventional transmission is about $6.89 \mathrm{bps} / \mathrm{Hz}$ (or 7.29 bps $/ \mathrm{Hz}$ ) when $\sigma_{\epsilon}^{2}=10^{-2}$, and this performance improvement will increase to about $8.76 \mathrm{bps} / \mathrm{Hz}$ (or $9.03 \mathrm{bps} / \mathrm{Hz}$ ) when $\sigma_{\epsilon}^{2}=10^{-3}$.

\section{CONCLUSiOnS}

This paper discussed the OFDMA uplink cooperative transmission in the presence of frequency offsets, with relays operating in either the AF or DF mode. By keeping the total transmit power of each symbol fixed, the outage information rate of each user was improved considerably. Since the accumulation of interference and noise in the relays in the AF mode degraded the effective SINR, the DF mode's cooperative transmission outperformed the AF mode with regards to the diversity gain, provided that an additional processing complexity was needed in the former and $\alpha$ is larger than a specified threshold. Numerical results illustrated the superior performance of the proposed cooperative relaying scheme over the conventional transmission with regard to outage information rate.

\section{REFERENCES}

[1] I. Koffman and V. Roman, "Broadband wireless access solutions based on OFDM access in IEEE 802.16," IEEE Commun. Mag., vol. 40, no. 4, pp. 96-103, Apr. 2002.

[2] C. Pan, Y. Cai, and Y. Xu, "Adaptive subcarrier and power allocation for multiuser MIMO-OFDM systems," in Proc. IEEE Int. Conf. Communications (ICC), vol. 4, May 2005, pp. 2631-2635.
[3] J. N. Laneman and G. W. Wornell, "Distributed space-time-coded protocols for exploiting cooperative diversity in wireless networks," IEEE Trans. Inform. Theory, vol. 49, no. 10, pp. 2415-2425, Oct. 2003.

[4] R. U. Nabar, H. Bolcskei, and F. W. Kneubuhler, "Fading relay channels: performance limits and space-time signal design," IEEE J. Select. Areas Commun., vol. 22, no. 6, pp. 1099-1109, Aug. 2004.

[5] B. Can, H. Yomo, and E. De Carvalho, "Hybrid forwarding scheme for cooperative relaying in OFDM based networks," in Proc. IEEE Int. Conf. Communications (ICC), vol. 10, Istanbul, June 2006, pp. 4520-4525.

[6] D. Sreedhar and A. Chockalingam, "ICI-ISI mitigation in cooperative SFBC-OFDM with carrier frequency offset," in IEEE Int. Symposium on Personal, Indoor and Mobile Radio Commun. (PIMRC), Athens, Sept. 2007, pp. 1-5.

[7] O. S. Shin, A. M. Chan, H. T. Kung, and V. Tarokh, "Design of an OFDM cooperative space-time diversity system," IEEE Trans. Veh. Technol., vol. 56, pp. 2203-2215, July 2007.

[8] T. C.-Y. Ng and W. Yu, "Joint optimization of relay strategies and resource allocations in cooperative cellular networks," IEEE J. Select. Areas Commun., vol. 25, no. 2, pp. 328-339, Feb. 2007.

[9] J. G. Proakis, Digital Communications, 4th ed. McGraw-Hill, 2001. 